\title{
HISTERECTOMÍA RADICAL EN EL HOSPITAL DE SAN JOSE 2000-2005
}

Edmundo Mora, MD*, Diana Marcela Novoa, MD.**

\section{Resumen}

Se presentan 16 mujeres con cáncer de cérvix (CCU) estadio IB1, a quienes se les practicó histerectomía radical modificada tipo Wertein más linfadenectomía pélvica bilateral como tratamiento de primera línea, evaluadas en el servicio de oncología ginecológica del Hospital San José de Bogotá (HSJ) entre los años 2000 y 2005 . Se determinó la morbilidad del procedimiento, definida como las complicaciones médicas o quirúrgicas derivadas del mismo, y se correlacionó con las variables edad, antecedentes médicos, tipo histológico, sangrado transoperatorio, duración del procedimiento, estancia hospitalaria, necesidad de tratamiento complementario, seguimiento y recaída.

Palabras clave: histerectomía radical, carcinoma del cérvix uterino.

\section{Introducción}

El CCU es una de las neoplasias más frecuentes a nivel mundial. En Colombia es la más común y representa el $26.8 \%$ de los cánceres en la mujer. ${ }^{1}$ En naciones avanzadas, lo usual es la detección de lesiones neoplásicas en etapas tempranas, al contrario de lo que sucede en los países en desarrollo, donde se diagnostican lesiones en estadíos avanzados. Poco más de 100 años han transcurrido desde que Wertheim realizara en Viena la primera histerectomía radical abdominal con linfadenectomía parcial. Fue hasta principios de los años 30 cuando Meigs modifica la técnica al realizar la linfadenectomía pélvica bilateral con una extensión mayor, convirtiéndose en el procedimiento estándar que efectuamos hasta la fecha. ${ }^{2}$ La mayoría de los estudios coinciden en que aquellas pacientes con $\mathrm{CCU}$ en etapas clínicas IB1 hasta IIA, tanto la radioterapia como la cirugía radical ofrecen resultados similares en supervivencia, cuyos rangos oscilan entre el 78 y 91\%. Sin embargo, las indicaciones precisas y el tipo de complicaciones difieren. ${ }^{2,3,4}$ En este artículo se

Fecha recibido: 10 de octubre de 2006 - Fecha aceptado: 27 de noviembre de 2006

* Ginecólogo oncólogo, Hospital de San José e Instituto Nacional de Cancerología, Bogotá, D.C, Colombia.

** Residente IV ginecología y obstetricia, Fundación Universitaria de Ciencias de la Salud, Hospital de San José, Bogotá D.C, Colombia. describe la experiencia en el servicio de oncología del departamento de ginecología y obstetricia del HSJ.

\section{Materiales y métodos}

Es un estudio descriptivo retrospectivo tipo serie de casos en donde se revisaron todos los casos de CCU atendidos durante el período comprendido entre el año 2000 y el 2005 en el HSJ. Se detectaron 16 pacientes con diagnóstico de neoplasia cervical estadío IB1 y que fueron llevadas a histerectomía radical modificada tipo Wertheim, más linfadenectomía pélvica bilateral como tratamiento de primera línea. Se excluyeron las pacientes que presentaron enfermedad extrapélvica o cuya clasificación clínica no coincidiera con estadios tempranos para ser sometidas a cirugía.

Todos los procedimientos fueron llevados a cabo por el servicio de oncología y la estadificación clínica fue realizada de acuerdo con la clasificación de la Federación Internacional de Ginecología y Obstetricia (FIGO) de 1995. El abordaje quirúrgico fue mediante una incisión media infraumbilical y revisión sistemática de la cavidad abdominal. La biopsia paraaórtica se realizó como procedimiento de rutina y la linfadenectomía pélvica incluyó los siguientes grupos ganglionares: ilíacos externos e 
internos, fosa obturadora y parametriales; la arteria uterina fue ligada en su origen de la hipogástrica y en todos los casos se preservó la arteria vesical superior. Se disecó el uréter en el parametrio (túnel de Meigs) para obtener un margen parametrial adecuado y disminuir la incidencia de fístulas.

\section{Resultados}

De las pacientes atendidas en el servicio de oncología durante el período del año 2000 al año 2005, 16 tuvieron diagnóstico de $\mathrm{CCU}$, se clasificaron en estadío IB1 y se llevaron a histerectomía radical modificada más linfadenectomía bilateral. Las características sociodemográficas de la población se resumen en la Tabla 1.

\begin{tabular}{|l|c|}
\hline \multicolumn{2}{|c|}{$\begin{array}{c}\text { Tabla I. Características sociodemográficas de la } \\
\text { población }\end{array}$} \\
\hline Parámetro & Media(años) \\
\hline Edad & $42,5(29-67)$ \\
\hline Menarquia & $14(12-17)$ \\
\hline Planificación & Negativa \\
\hline $\begin{array}{l}\text { Inicio de relaciones } \\
\text { sexuales }\end{array}$ & $18(18-30)$ \\
\hline $\begin{array}{l}\text { Número de compañeros } \\
\text { sexuales }\end{array}$ & $(2-4)$ \\
\hline Primer parto & $19(16-23)$ \\
\hline
\end{tabular}

El diagnóstico fue hecho por citología sospechosa, colposcopia y biopsia en $66 \%$ de las pacientes, en $26 \%$ se realizó en la conización al observar displasia no graduable en la primera biopsia y en una se llegó al diagnóstico por legrado fraccionado. El tamaño tumoral osciló entre lesiones no visibles hasta $4 \mathrm{~cm}$.

La histología preoperatoria predominante fue carcinoma escamocelular en ocho casos (53.3\%) y en siete adenocarcinoma (46,6\%). El 80\% fueron remitidas al servicio de oncología de otras instituciones prestadoras de salud (IPS) y se hizo el diagnóstico en la unidad de patología cervical del Hospital de San José en 20\%.

En cuanto al transoperatorio, no se evidenciaron lesiones extrapélvicas en ninguna paciente, ni hubo complicaciones intraoperatorias. En las pacientes premenopáusicas se preservó la función ovárica, en todas se dejó dren de penrose por vía vaginal y se utilizó sonda Foley. El tiempo promedio de cirugía fue de tres horas (rango de una a cuatro horas) y el sangrado transoperatorio promedio varió entre 500 y $1000 \mathrm{cc}$. en el $60 \%$ de las pacientes. La estancia hospitalaria fue de tres días en el $86 \%$ de las pacientes y la más prolongada correspondió a una enferma que tuvo perforación del yeyuno al quinto día postoperatorio y desarrolló pelviperitonitis por lo que requirió manejo en UCI por 15 días, con evolución posterior favorable.

Una paciente desarrolló íleo postoperatorio (6\%), que se resolvió con reposo intestinal y corrección hidroelectrolítica, otra $(6 \%)$ acusó infección de la cúpula vaginal que cedió con la administración de antibióticos y una tercera presentó fístula vesicovaginal corregida con la intervención posterior del departamento de urología (6\%).

Los hallazgos histopatológicos definitivos fueron los siguientes: carcinoma escamocelular seis casos $(40 \%)$; adenocarcinoma cuatro ( $26 \%$ ); adenoescamoso uno $(6 \%)$; mixto uno $(6 \%)$ y negativo para neoplasia residual dos pacientes (13\%). El número de ganglios pélvicos disecados fue en promedio 16 (rango de 4 a 38), una paciente presentó metástasis a cinco ganglios ilíacos e invasión vascular por lo cual requirió tratamiento adyuvante con radioterapia. Se encontró compromiso mayor del 50\% del cuello en el $53 \%$ de las pacientes.

En ocho casos se utilizó tratamiento adyuvante con base en radioterapia y las indicaciones para su realización fueron compromiso cervical mayor del $50 \%$, invasión vascular y compromiso ganglionar.

En promedio el tiempo de seguimiento sin pérdida de las pacientes por remisión a otras instituciones fue de seis meses, sin encontrar casos de recidiva tumoral. 
La sobrevida no es posible estimarla ya que hay una deserción importante por causas administrativas. Sin embargo, las que continúan en controles tienen una supervivencia del 95\%.

\section{Discusión}

El tratamiento de elección en pacientes con estadios tempranos de CCU (IA2 y IB1) es la histerectomía radical. La razón tradicional para elegirla sobre la radioterapia se centra en la edad de la enferma y el deseo de preservar la función ovárica. Quizás representan contraindicaciones relativa s la obesidad, el riesgo cardiovascular y la posibilidad de complicaciones como la estenosis vaginal. ${ }^{3,5}$

En la primera mitad del siglo pasado para todas las etapas de la enfermedad el tratamiento básico del CCU consistía en radioterapia, dada la elevada tasa de mortalidad quirúrgica. En la actualidad la mayor destreza y experiencia ganada por los cirujanos, los avances en antibioticoterapia y en anestesia, así como el desarrollo de las unidades de cuidados intensivos postoperatorios, han reducido el número de muertes por cirugía y hoy día fluctúa entre 0 y $4.7 \%$. ,6

En tanto que la histerectomía radical permite conservar la función ovárica y minimiza el deterioro de la función sexual, la radioterapia puede condicionar estenosis o resequedad vaginal, dispareunia, cistitis y proctitis en menor o mayor grado. Además, mediante la cirugía es posible obtener la pieza quirúrgica para la evaluación histopatológica del tumor primario y ganglionar con fines pronósticos y de decisión del manejo adyuvante, pero fundamentalmente el estatus ganglionar, de donde en presencia de ganglios negativos la supervivencia a cinco años es de alrededor del $90 \%$ y con ganglios positivos se reduce al 50-65\%. $3,4,6,7$

El tratamiento quirúrgico radical no está exento de complicaciones. El riesgo es mayor si se combina la cirugía con la radioterapia. La incidencia de infección pélvica sin antibioticoterapia profiláctica oscila entre 2 y $36 \%$, que al compararse con nuestro estudio, a quienes sí se les aplicó, revelan un menor porcentaje de infección en nuestros casos $(6 \%)$. La infección de la herida varía entre el 3 y el $47 \%$. $^{5,7}$ Las fístulas del tracto urinario, disfunciones vesicales neurogénicas, linfoquistes, hemorragias y los eventos tromboembólicos son considerados como complicaciones principales tras la histerectomía radical, con una incidencia del 3 al 16\%; solo una de las pacientes intervenidas ( $6 \%$ ) desarrolló como complicación fístula vesicovaginal, con posterior resolución del cuadro.

En etapa clínica IB1, el manejo quirúrgico con histerectomía radical puede ser la única modalidad de tratamiento, ya que en estadíos IB2 y IIA la posibilidad de manejo combinado es mayor.

La distribución de los tipos histológicos registrada en nuestra serie es muy similar a la consignada en la literatura, y la distribución por edad y características sociodemográficas coinciden con los hallazgos de mayor predisposición del CCU en grupos de mujeres con menarquia o inicio de relaciones sexuales tempranas, primer parto antes de $\operatorname{los} 17$ años y multiparidad. ${ }^{3,4}$

El tiempo quirúrgico promedio y el sangrado transoperatorio se encuentran dentro de los parámetros permitidos observados en la literatura. La cifra de ganglios pélvicos positivos en estadío IB es del $20 \%{ }^{3}$ Sin embargo, en nuestros hallazgos solo encontramos una paciente $(6 \%)$ con cinco ganglios ilíacos positivos que fue enviada a radioterapia complementaria.

El principal factor determinante del éxito en la cirugía de CCU es el tamaño de la lesión. Ejemplo de este hallazgo es lo demostrado por Piver y colaboradores, los cuales lograron una supervivencia a cinco años del $90 \%$ de aquellos con tumores hasta $3.0 \mathrm{~cm}$. y ausencia de enfermedad metastásica ganglionar, significativamente mejor al 16\% de quienes tenían lesiones mayores a $3.0 \mathrm{~cm}$. y/o ganglios pélvicos positivos. La sola presencia de ganglios pélvicos positivos resultó predictor para recurrencia, demostrado en ambos análisis ( $\mathrm{p}<0.001)$; sin importar el estado ganglionar, 13 pacientes en su estudio recurrieron localmente y sólo una a distancia, lo que confirma 
que existe un subgrupo de enfermas de alto riesgo para recaída local o regional con ganglios negativos, en quienes deben adoptarse conductas terapéuticas más agresivas o aplicarse otros métodos predictores de recurrencia. En nuestro estudio a los seis meses de seguimiento no se presentó ninguna recaída.

El tratamiento quirúrgico ofrece ventajas sobre la radioterapia, entre ellas el conocimiento del estado ganglionar y las tasas de supervivencia en pacientes con ganglios negativos que nos revelan $90 \%$ de supervivencia. ${ }^{8}$

La histerectomía radical es un procedimiento quirúrgico cuyos criterios estrictos en la selección de pacientes ofrece una cifra de curación del $90 \%$ y en centros especializados un bajo porcentaje de complicaciones.

\section{Referencias}

1. Molano M, van den Brule AJ, Posso H, et al. Low grade squamous intra-epithelial lesions and human papillomavirus infection in colombian women. Br J Cancer. 2002; 87(12):1417-21.

2. Selman TJ, Luesley D.M. Is radical hysterectomy for early stage cervical cancer an outdated operation?. BJOG. 2005; 112: $363-65$.

3. Symmonds, R. Morbidity and complications of radical hysterectomy with pelvic lymph node dissection. Am J Obstet Gynecol. 1996; 94: 663-73.

4. Solorza L, Lobatón A. Histerectomía radical como tratamiento del cáncer en cuello uterino. Experiencia interinstitucional en México. Gamo. 2004; 3 (4): 31-34.

5. Esquivel G, Rodríguez F. Histerectomía Radical. Rev Inst Nal Cancerol (Mex). 2000; 46 (2): 100-02.

6. Jackson KS, Pelvic floor dysfunction and radical hysterectomy. Int J Gynecol Cancer. 2006; 16: 354-63.

7. Bosque D, Arrebola J, Iruretagoyena B. Histerectomía radical en cáncer cervicouterino en estadios IA2-IB. Rev Cubana oncol. 1999; 15 (1):19-25.

8. Barragán C. López E. Factores pronóstico en pacientes con cáncer cervicouterino epidermoide tratadas con histerectomía radical. Rev Inst Nal Cancerología (Mex). 1999; 45 (4).

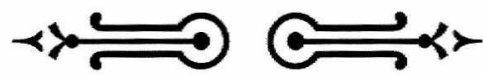

